\title{
Neural Network-Based Model for Joint Prediction of the Newtonian and NonNewtonian Viscosities of Black Liquor
}

\author{
S. B. Alabi and C. J. Williamson
}

\begin{abstract}
Most of the existing models for describing black liquor (BL) viscosity behaviours are applicable over limited ranges of process conditions, whereas BL exhibits varied viscosity behaviours, Newtonian and nonNewtonian, over a wide range of process conditions. These limited-range models, resulting from different bases, may suffer predictions continuity over such wide ranges of conditions. In this paper, attempt was made to jointly model the Newtonian and nonNewtonian viscosity behaviours of literature liquor using artificial neural network (ANN) paradigm. A generalized multilayer feedforward network with 7 hidden neurons and 1 output neuron, having $R^{2}=1.0$ and maximum absolute relative error of $\sim 8 \%$ between the actual and predicted data was obtained. Although a model with a higher accuracy is desirable, the proposed single network seems to be a reasonable alternative to the use of the limited-range multiple models for the purposes of describing black liquor viscosity behaviour over a wide range of practical conditions.
\end{abstract}

Index Terms-Artificial neural network, black liquor, Newtonian, NonNewtonian, viscosity.

\section{INTRODUCTION}

Kraft black liquor (BL) is a by-product of kraft pulping process. The liquor viscosity plays significant roles in the performance of kraft recovery facilities in a pulp and paper mill. Its viscosity affects the heat transfer in the evaporation units, capacity of pumps and size of black liquor droplets being sprayed in the recovery boiler (RB) [1], [2]. Black liquor exhibits varied viscosity behaviours over a wide range of process conditions. It is approximately Newtonian at low solids concentrations (SCs) but shows nonNewtonian behaviour at high SCs [3]. Under certain circumstances, it shows Newtonian behaviour at high SCs and exhibits nonNewtonian behaviour at low SCs [4]. Availability of accurate and reliable models which are capable of describing the viscosity behaviours of black liquor over a wide range of process conditions is therefore essential for the improvement in the design, analysis and operation of kraft recovery facilities.

Majority of the literature models for predicting black

Manuscript received May 24, 2014; revised September 28, 2014. This work was financially supported by the Foundation for Research, Science and Technology (Grant: CHHP0601), Carter Holt Harvey Pulp and Paper (CHHP\&P) (at Kinleith) and Department of Chemical and Process Engineering, University of Canterbury, New Zealand.

S. B. Alabi is with the Department of Chemical and Petroleum Engineering, University of Uyo, Uyo, Nigeria (e-mail: sundayalabi@uniuyo.edu.ng).

C. J. Williamson is with the Department of Chemical and Process Engineering, University of Canterbury, Christchurch, New Zealand (e-mail chris.williamson@canterbury.ac.nz). liquor viscosity are applicable at limited ranges of process conditions. The study of black liquor viscosity behaviours over a wide range of process conditions may therefore require the use of multiple models. Unfortunately, this may pose the challenge of predictions continuity, as individual limited-range models were developed from different bases. A promising approach would be to use a single model derived from a wide range of process conditions. The lowshear Newtonian viscosity functional models reported in [5]-[8] and which are applicable at all solids concentrations seem to be promising. Unfortunately, the ranges of applicable temperatures are unspecified for the models reported in [5], [6]. Vakkilainen's [8] model is applicable over only a very narrow range of temperatures close to actual operating conditions. Ghosh's [7] model has been shown to be ineffective for predicting black liquor viscosity even at limited low-solids concentrations [9]. The recently proposed artificial neural network (ANN)-based model for predicting the viscosity of black liquor is applicable over a wide range of process conditions [10]. However, it is limited to Newtonian black liquors. Zaman and Fricke [11], [12] proposed two models(modified Cross and CarreauYasuda) for predicting the viscosity of nonNewtonian softwood kraft black liquor. One of the downsides of these models is that they are only applicable at high solids concentrations $(\mathrm{SC}>50 \%)$. Under certain circumstances, black liquor shows Newtonian behaviour at high SCs and exhibits nonNewtonian behaviour at low SCs [4]. It is therefore essential to have a model which is capable of jointly describing the Newtonian and nonNewtonian behaviour of black liquor over a wide range of process conditions. Hitherto, such model has not been reported in the literature. In this paper, artificial neural network paradigm was attempted to jointly model the Newtonian and nonNewtonian behaviour of black liquor over a wide range of process conditions. The predictive and generalization capacities of the developed models were tested using limited experimental data and response surface methodology (RSM).

\section{BLACK LIQUOR VISCOSITY MODELING}

\section{A. Data Collection}

The black liquor viscosity data utilized for the development of ANN-based models described in this paper were obtained from the literature (see Fig. 2 in [4]). The detailed step by step approaches adopted to measure the viscosities of the liquor samples, as a function of its influencing variables have been published in [4]. The data 
ranges are as follows: solids concentration (SC), $25-70 \%$, temperature $(\mathrm{T}), 0-115^{\circ} \mathrm{C}$, shear rate, $\sim 10-2000 \mathrm{~s}^{-1}$ and viscosity, $\sim 1-6300 \mathrm{mPa}$.s. Since solids concentration of the weak black liquor is usually around $14-18 \%$ when it leaves the digester or the pulping unit [13], black liquor viscosity data at solids concentrations $<25 \%$ would be required to develop models that cover all practical ranges of solids concentration. Therefore, viscosity data for black liquor at solids concentration of $0 \%(100 \%$ pure water $)$ were obtained over

$$
\begin{aligned}
& \mu_{w}(\mathrm{mPa} . \mathrm{s})=0.355-0.2053 z+0.1006 z^{2}-0.0518 z^{3} \\
& +0.02461 z^{4}-0.001536 z^{5}-0.0006853 z^{6} \\
& -0.003967 z^{7}+0.001774 z^{8}
\end{aligned}
$$

the temperature range $0-160{ }^{\circ} \mathrm{C}$ from the database of the physical properties of water provided by ThermExcel [14].

The water viscosity $\left(\mu_{w}\right)$ data were then fitted to the model given in (1), where $z=(T-80) / 46.62 ; T=$ temperature $\left({ }^{0} \mathrm{C}\right)$. The performance measures of $\mu_{w}$ are: $R^{2}=1.0$ and MARE (maximum absolute relative error) $=0.71 \%$. Equation (1) was used to estimate the viscosity of water (or black liquor at solids concentration $=0 \%$ ) where necessary.

At low solids concentrations $(<=50 \%)$, black liquor traditionally exhibits Newtonian behaviour. The liquor considered in this study, up to around $51 \%$ and at all temperatures, is a Newtonian or nearly Newtonian fluid. At higher solids concentrations (> $51 \%$ ), it shows shearthinning behaviour at some process conditions. The variations of the viscosity of a shear-thinning fluid will often reach a limiting value at very low shear rates or at high shear rates where it behaves typically as Newtonian fluid. The limiting viscosity at low shear rates is termed zero shear viscosity, $\mu_{\infty}$ while the limiting viscosity at very high shear rates is termed infinite shear viscosity, $\mu_{\infty}$ [15]. In order to obtain complete data for the development of a single model which is capable of jointly describing the Newtonian and nonNewtonian behaviours of black liquor over a wide range of process conditions, the following steps were taken. First, the liquor nonNewtonian viscosities were approximated by their Newtonian equivalents at low shear rates (shear rate $\sim=0$ ). Cross model [16] given in (2) was used to estimate zero shear viscosity of black liquor (see [17]) from the viscosity-shear rate data obtained at the easily measurable shear rates. $\gamma$ is the shear rate $\left(\mathrm{s}^{-1}\right), \mathrm{C}$ is the Cross time constant(s) and $\mathrm{m}$ is the dimensionless Cross rate constant [18].

$$
\mu=\mu_{\infty}+\frac{\mu_{0}-\mu_{\infty}}{1+(C \gamma)^{m}}
$$

In practice, $\mu_{\infty}$ may not be attained and so is deemed to be negligible when compared with zero-shear viscosity and viscosities at other measurable shear rates. Setting $\mu_{\infty}=0.0$ in (2) and using the nonlinear curve-fitting tools in the MATLAB $^{\circledR}$ optimization toolbox, $\mu_{\infty}$ ranging from $\sim 12360 \mathrm{mPa}$.s to $12780 \mathrm{mPa}$.s were obtained from the available shear-dependent viscosity data of the chosen literature liquor. Secondly, the zero-shear viscosity data obtained above were combined with the average viscosity data where the liquor exhibits pure Newtonian or nearNewtonian behaviour and, the viscosities of pure water (black liquor at $0 \%$ solids concentration) to obtain Newtonian viscosity data over a wide range of process conditions. It should be noted that in the ANN-based modelling, the input and response variables data are normally pre-processed so that all data fall within $[-1,1]$ or $[0,1]$ to enhance neural network training [19], [20]. When the pattern in the data is exponential or in the power law form, it is advisable that they are log-transformed before applying the mapping or scaling functions [19]. Consequently, at zero-shear conditions i.e. where shear rate tends to zero, $\log (0)$ is undefined for the scaling process mentioned above. In order to be able to define low-shear conditions for ANN modelling, it was assumed that shear $\operatorname{rate}\left(\mathrm{s}^{-1}\right)$ of $10^{-4} \sim=0$. So, low shear rate was set equal to $10^{-}$ ${ }^{4} \mathrm{~s}^{-1}$. Finally, the Newtonian viscosity data (original, pure water and zero-shear) obtained above were combined with the original shear-dependent data of the literature liquor to obtain combined Newtonian and nonNewtonian viscosity data comprising of solids concentration, 0-70\%, temperature, $0-115^{\circ} \mathrm{C}$, shear rate, $\sim 0.0001-2000 \mathrm{~s}^{-1}$, and viscosity, $\sim 1-$ $12780 \mathrm{mPa}$.s. Flowing black liquors typically have shear rates of 30-4000 $\mathrm{s}^{-1}$ [5] and Chhabra and Richardson [21] have shown that many materials attain $\mu_{0}$ at shear rates $\leq$ $0.01 \mathrm{~s}^{-1}$. Therefore taking $10^{-4} \mathrm{~s}^{-1}$ as the low shear rate value is justified for black liquor processing.

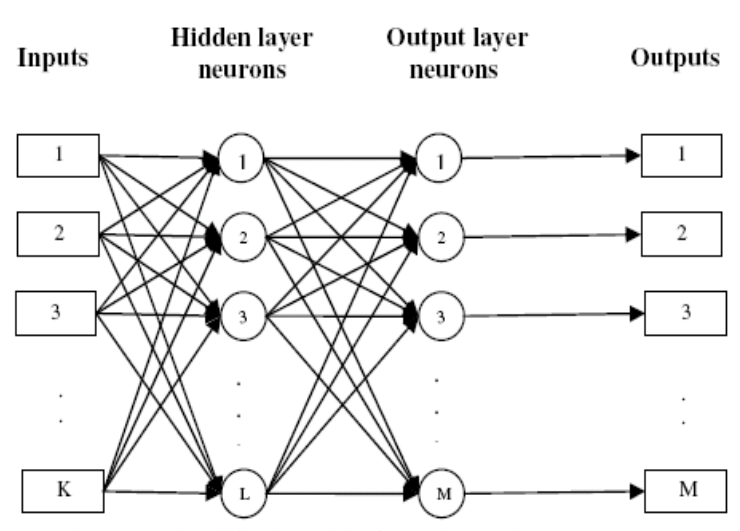

Fig. 1. A simplified model of a typical 2-layer FF ANN.

\section{B. Black Liquor Viscosity Model Using Artificial Neural Network (ANN) Paradigm}

ANN is the generalization of the mathematical model of a biological nervous system [22]. It is a highly parallel system that processes information through modifiable weights, thresholds/biases, and mathematical transfer functions [23]. Different architectures for, and training algorithms associated with ANNs are well discussed in the literature (e.g. [20]-[25]). This paper describes only the application of multi-layer (ML) feed-forward (FF) ANN to the modelling of $\mathrm{BL}$ viscosity, as a function of its chosen influencing variables-solids concentration, temperature and shear rate. In the FF networks, the signal flow is from the input to the output units, strictly in a feed-forward direction. The data processing can extend over one or several layers (ML) of neurons, but no feedback connections are present [22]. A simplified model of a typical 2-layer FF ANN is given in Fig. 1. In this work, the hyperbolic tangent sigmoid transfer 
function and a linear transfer function, as shown in Fig. 2, were used in the hidden and output neurons, respectively. In this paper, a network with 1 hidden layer (having $\mathrm{L}$ neurons) and 1 output layer (having $M$ neurons) is denoted as an $[\mathrm{L}, \mathrm{M}]$ network while a network with 2 hidden layers (having $\mathrm{K}$ neurons in the first hidden layer and $\mathrm{L}$ neurons in the second hidden layer) and 1 output layer (having $M$ neurons) is denoted as a $[\mathrm{K}, \mathrm{L}, \mathrm{M}]$ network.
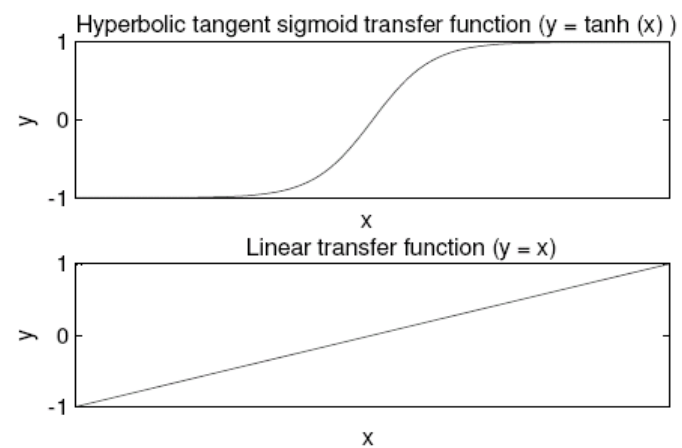

Fig. 2. Transfer functions used in this work.

\section{ANN Training and the Criteria for the Evaluation of the Goodness of the Resulting Networks}

The inputs to the networks are solids concentration, temperature and shear rate while the only output is viscosity. Prior to the network's training, the input and the output variables were pre-processed to fall in the range $[-1,1]$ (see [26]). The solids concentration was expressed in a fractional form (0-1) while temperature was expressed in Kelvin. The shear rate $\left(\mathrm{s}^{-1}\right)$ and the viscosity (mPa.s) were logtransformed prior to being scaled to within $[-1,1]$. The steps described in [26] for training ANN via Bayesian regularization algorithm available in the $\mathrm{MATLAB}^{(\mathrm{R})}$ neural network toolbox, were followed to arrive at the networks reported in this paper. The typical criteria for the evaluation of the goodness of a Bayesian-trained network include Marquardt adjustment parameter (MU), the effective number of parameters per total number of tunable parameters (\#), sum of squared weights (SSW), and the network prediction error. It is important to train the network until the network parameters converge. Given a sufficient training epochs (depending on the complexity of the problem) e.g. 5000, a true convergence is attained when maximum MU is reached. Convergence is also attained when \#, SSW, and the network prediction error remains fairly constant over a significant number of iterations [19]. In addition to monitoring the above-stated indices, the goodness of the ANNs developed in this study were examined based on two other performance criteria namely coefficient of determination, $R^{2}$ and maximum absolute relative error (MARE). $R^{2}$ is the square of correlation coefficient $(R)$ between the model predictions and experimental observations. $R^{2}=0$ indicates a poor model while $R^{2}=1$ indicates a very good model. However, a model with a high $R^{2}$ may not necessarily be a very good model [27], most especially where the fitted model involves transformation/scaling of the response variable(s). There is no guarantee that when the model predictions are translated to the actual scale that the level of accuracy attained in the transformed state will also be attained in the untransformed state. Therefore, MARE (\%)), as given in (3), was used in addition to $R^{2}$ to evaluate the goodness of the ANN-based models described in this paper:

$$
\operatorname{MARE}(\%)=100 \operatorname{maximum}\left(\left|\frac{\mu_{p}-\mu_{a}}{\mu_{a}}\right|\right)
$$

where $\mu_{p}=$ predicted viscosity (mPa.s) in an untransformed state, $\mu_{a}=$ actual viscosity (mPa.s) in an untransformed state, $|\cdot|=$ modulus (absolute value) of the terms in the bracket. Once a network which satisfies all these conditions is found, the training can be terminated. A model with a very low MARE, if possible, $0 \%$ is desirable.

\section{RESUlTS AND DISCUSSION}

Following the ANN training procedures described in section 2.3, different ANN structures and sizes were searched. Although in all cases, maximum MU was attained, true convergence in terms of \#, SSW and SSE was not attained. For example, for 1-hidden layer network, increasing the number of hidden neurons from 7 to $10 \mathrm{did}$ not bring any appreciable difference in the $R^{2} s$ (all = 1) and MAREs (decrease from 8.1 to $6.3 \%$ ) of the networks. However, \# increases substantially from 33 to 45 and SSW decreases noticeably from 265.13 to 173 . Therefore, a [7, 1] network was selected pending further performance evaluation tests. Although other networks having hidden neurons, \#s and SSWs (except the network with 1 hidden neuron) less than that of $[7,1]$ network were obtained, but they were less accurate in terms of MAREs $(15 \%-105 \%$ compared to $8.1 \%$ )) despite having $R^{2} s>0.98$. Since the overall target was to have a network with $R^{2}=\sim 1$ and MARE as low as possible, the search was terminated when a network having 11 hidden neurons with $\#=52$; SSW=481.4; $R^{2}=1.0$ and MARE $=3.38 \%$ was obtained. Attempt was also made to search for a 2-hidden layer network to model the entire data. The nonconvergence trend similar to the one observed while training the 1-hidden layer network was also observed. Therefore, the training was terminated when a [4, $4,1]$ network having $\#=38, \mathrm{SSW}=183, \mathrm{MARE}=4.7 \%$ and $R^{2}=1.0$ was obtained.

A possible factor responsible for the lack of true convergence to a unique solution is that the ANN may be having difficulty learning the relationship between black liquor viscosity and the three input variables, solids concentration, temperature and shear rate, under the conditions where shear rate does not affect the liquor viscosity. At solids concentrations $<=50.98 \%$, the viscosity of the literature liquor is independent of shear rate. At higher solids concentrations ( $>50.98 \%$ ), the liquor viscosity is independent of shear rate at high temperatures; however, at lower temperatures and/or high shear rates, the liquor shows shear-dependence behaviour [4]. It is very likely that the ANNs see shear rate as a redundant variable under the Newtonian conditions and therefore, this made its learning difficult. While it is clear that at the Newtonian regions, black liquor viscosity is independent of shear rate, this is not so at the nonNewtonian regions. Despite suffering redundancy problem, it is clear that ANN is potentially able 
to accurately model the joint Newtonian and nonNewtonian behaviours of black liquor over a wide range of process conditions. A network with internal classification ability (beyond the scope of this current study), trained using other algorithms, may be more effective than multilayer feedforward ANN.

TABLE I: PRELIMINARY VALIDATION DATA FOR THE LITERATURE LIQUOR

\begin{tabular}{lccc}
\hline SC $(\%)$ & Temperature $\left({ }^{\circ} \mathrm{C}\right)$ & Shear rate $\left(\mathrm{s}^{-1}\right)$ & Viscosity $(\mathrm{mPa} . \mathrm{s})$ \\
\hline 11.95 & 16 & 1000 & 2.138 \\
11.95 & 16 & 1500 & 2.138 \\
11.95 & 16 & 2000 & 2.138 \\
11.95 & 25 & 1000 & 1.773 \\
11.95 & 25 & 1500 & 1.773 \\
11.95 & 25 & 2000 & 1.773 \\
31.85 & 10 & 200 & 10.755 \\
31.85 & 10 & 400 & 10.755 \\
31.85 & 30 & 500 & 5.615 \\
31.85 & 30 & 1000 & 5.615 \\
31.85 & 30 & 1500 & 5.615 \\
31.85 & 30 & 2000 & 5.615 \\
\hline
\end{tabular}

$\mathrm{SC}=$ solids concentration.

TABLE II: PERFORMANCES OF THE ANN-BASED VISCOSITY MODELS FOR THE LITERATURE LIQUOR OVER A WIDE RANGES OF PROCESS CONDITIONS

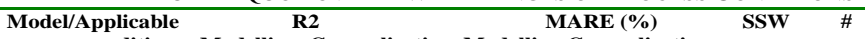
\begin{tabular}{lllllll} 
process conditions & Modelling & Generalization & Modelling Generalization & \\
\hline ANN [7,1] & 1.0 & 0.9943 & 8.08 & 6.45 & 265.13 & $33 / 36$
\end{tabular} @ all SR, all T

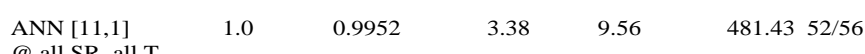

$\begin{array}{lllllll} & & & & & & \\ \text { ANN }[4,4,1] & 1.0 & 0.9997 & 4.70 & 13.73 & 183.10 & 38 / 41\end{array}$

(i) all SR, all T

( $\mathrm{SR}=$ shear rate and $\mathrm{T}=$ temperature)

TABLE III: INTERMEDIATE NON-NEWTONIAN VISCOSITY DATA FOR THE LITERATURE LIQUOR

\begin{tabular}{|c|c|c|c|c|c|}
\hline \multirow[b]{2}{*}{$\operatorname{sC}(\%)$} & & \multirow[b]{2}{*}{ ANN $[4,4,1]$ predictions (mPa.s $)$} \\
\hline & Temperature $\left({ }^{\circ} \mathrm{C}\right)$ & Shear rate $\left(\mathrm{s}^{-1}\right)$ & ANN [7 1] predictions (mPas) & ANN $[11,1]$ predictions $\left(\mathrm{mPa}^{\mathrm{a}}\right)$ & \\
\hline 0 & $90^{*}$ & 10 & 0.3155 & 0.3153 & 0.3148 \\
\hline $60^{*}$ & 60 & 10 & 291.3 & 284 & 352.5 \\
\hline $61^{*}$ & 40 & 10 & 2257 & 2563 & $281^{* *}$ \\
\hline $66^{\circ}$ & 80 & 100 & 396.6 & 469.4 & 544.5 \\
\hline $54^{*}$ & 40 & 100 & 153.6 & 134.1 & 135.2 \\
\hline $10^{*}$ & 0 & 1000 & 3.559 & 2.344 & 2599 \\
\hline $30^{*}$ & 40 & 1000 & 3.475 & 3.409 & 3.687 \\
\hline $45^{*}$ & 40 & 1000 & 17.18 & 18.43 & 16.53 \\
\hline $5^{*}$ & 60 & 1000 & 0.6325 & 0.7531 & 0.6286 \\
\hline $28^{\circ}$ & 60 & 1000 & 2.106 & 2.153 & 2.214 \\
\hline $20^{*}$ & 80 & 1000 & 1.086 & 1.083 & 0.9788 \\
\hline $40^{*}$ & 80 & 1000 & 3.341 & 3.262 & 3.14 \\
\hline $53^{*}$ & 100 & 1000 & 10.53 & 10.94 & 11.03 \\
\hline $60^{*}$ & 100 & 1000 & 42.27 & 41.37 & 40.99 \\
\hline 37.09 & $30^{*}$ & 1500 & 8.769 & 8.863 & 8.825 \\
\hline 37.09 & $50^{*}$ & 1500 & 4.68 & 4.705 & 4.695 \\
\hline 58.56 & $95^{*}$ & 1500 & 37.1 & 36.85 & 37,02 \\
\hline 24.73 & $10^{*}$ & 2000 & 6.522 & 6.211 & 5.884 \\
\hline $30^{\circ}$ & 80 & 2000 & 1.774 & 1.707 & 1.828 \\
\hline $42^{*}$ & 80 & 2000 & 3.967 & 3.899 & 3.644 \\
\hline
\end{tabular}

"intermediate data point
" noticeably off prediction

\section{A. Preliminary Validation Test}

In order to ascertain the generalization ability of the three shear-dependent ANNs obtained above, they were exposed to the independent data in Table I. The performances of the networks are as shown in Table II. It is observed that $[7,1]$ network, the least accurate among the three networks, has a generalization error smaller than, but comparable (difference<2\%) with its modelling error. This indicates good generalization ability. Conversely, the two larger and more accurate networks have generalization errors that are roughly 3 times larger than their corresponding modelling errors, indicating that they have poor generalization abilities. On comparing the $[11,1]$ and $[4,4,1]$ networks, the more accurate [11,1] network, having larger SSW and \# than those of $[4,4,1]$ network would have been expected to generalize less than the $[4,4,1]$ network; but the generalization results proved otherwise. It seems logical to say that the better performance of the $[11,1]$ network over the $[4,4,1]$ network depends on its simpler (1-hidden layer) structure/configuration rather than the number of its parameters or the size of its SSW. Conversely, for the networks of the same configuration (1-hidden layer), the more accurate $[11,1]$ network with the larger SSW and more \#, is less generalized than the $[7,1]$ network, as expected. It seems logical to conclude that, the $[7,1]$ network's generalization ability is a function of its SSW. Although it was difficult to obtain a unique Bayesiantrained multi-layer feed-forward ANN-based model for predicting black liquor viscosity over a wide range of process conditions, it is evident that, for practical applications, the least accurate (with MARE=8.1\%), but most generalized (with MARE $=6.45 \%$ ) $[7,1]$ network obtained in this study, may be suitable.

\section{B. Generalization Performances of the Joint Newtonian and non-Newtonian Black Liquor Viscosity Models}

The generalization capabilities of the three ANN-based models which were developed for jointly predicting Newtonian and nonNewtonian viscosity of black liquor as a function of solids concentration, temperature and shear rate, over a wide range of process conditions, were evaluated using independent data points which were limited to low solids concentrations and viscosities where the liquor behaves typically as Newtonian fluid. The results showed that only $[7,1]$ network has good generalization capability under these conditions. Unfortunately, it is not clear whether the models will generalize well to new inputs at high solids concentrations and high viscosities where black liquor is known to typically exhibit nonNewtonian behaviour. To be able to investigate the models' predictive and generalization capabilities in the absence of experimental data under these conditions, response surface methodology (RSM) which was recently suggested in [10] was adopted. Following the procedures outlined in [10], these networks were therefore exposed to new input data sets-solids concentrations and temperatures at carefully selected intermediate values of the known modelling input data points for shear rates spanning more than three decades (Table III). The prediction surfaces, multiple three dimensional (3D) scatter plots of the modelling and intermediate outputs were obtained at each shear rate for the three networks . At $10 \mathrm{~s}^{-1}$, as observed from Fig. 3, the modelling outputs from all the three networks fall smoothly on their prediction surfaces, indicating that the models are able to reasonably predict their outputs from the known inputs. However, only the $[7,1]$ and $[11,1]$ networks are able to predict the intermediate outputs, which all fall on their respective surfaces at reasonable intermediate positions between the modelling outputs. The $[4,4,1]$ network with the most complex architecture among the three networks predicts very low viscosity of $281 \mathrm{mPa} . \mathrm{s}$ at solids concentration $=61 \%$ and temperature $=40 \quad{ }^{\circ} \mathrm{C}$, as opposed to viscosity $>2000 \mathrm{mPa}$.s predicted by $[7,1]$ and $[11,1]$ networks at the same conditions. Consequently, as shown in Fig. 3, only the predictions from [7, 1] and [11, 1] networks are large enough to be noticed on their respective 
surfaces. This result shows that $[4,4,1]$ network might have over-fitted its training data, and hence failed to generalize to new inputs at $10 \mathrm{~s}^{-1}$ and high viscosity. Following the procedures utilized at $10 \mathrm{~s}^{-1}$ the networks performances were also evaluated at $100 \mathrm{~s}^{-1}, 1000 \mathrm{~s}^{-1}, 1500 \mathrm{~s}^{-1}$ and $2000 \mathrm{~s}^{-}$ ${ }^{1}$ using the data in Table III. Fig. 4 shows the models generalization performances at $1000 \mathrm{~s}^{-1}$. At all process conditions, modelling and the intermediate outputs from all the three networks fall smoothly on their respective prediction surfaces while the intermediate outputs fall at reasonable intermediate positions between the modelling outputs. This finding demonstrates that all the networks give reasonable predictions and have strong generalization capabilities at $1000 \mathrm{~s}^{-1}$. The models' performance results at 100, 1500 and $2000 \mathrm{~s}^{-1}$ are found to be similar to their performances at $1000 \mathrm{~s}^{-1}$. It is concluded that over wide ranges of solids concentrations, temperatures, shear rates, and viscosities, [7, 1] and [11, 1] ANNs would give reasonable black liquor viscosity estimates when used with known and new input data. Since a model with a low complexity is usually desirable, a [7, 1] network is suggested for the prediction of the joint Newtonian and nonNewtonian viscosities of the literature liquor over a wide range of process conditions.

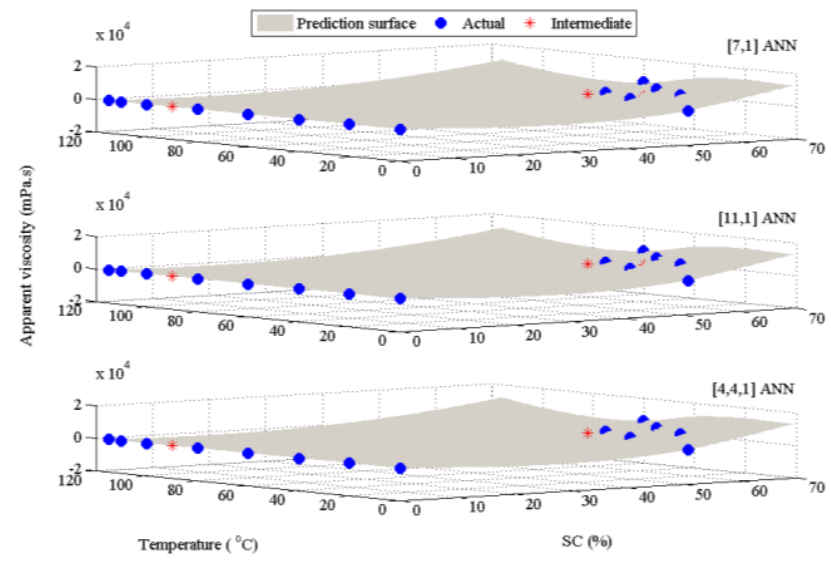

Fig. 3. Generalization performances of three ANN-based models for the literature liquor at $10 \mathrm{~s}^{-1} ;(\mathrm{SC}=$ solids concentration $)$.
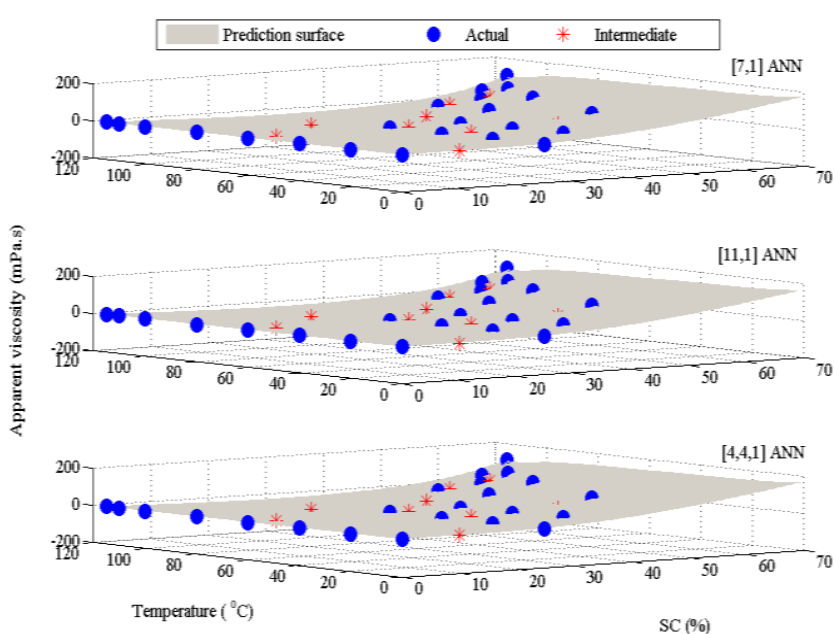

Fig. 4. Generalization performances of three ANN-based models for the literature liquor at $1000 \mathrm{~s}^{-1} ;(\mathrm{SC}=$ solids concentration $)$.

\section{CONCLUSIONS}

Hitherto, the available models describing black liquor viscosity behaviour were applicable over limited ranges of process conditions. This would require the use of multiple models for the analysis of its behaviour over wide ranges of conditions. This study developed artificial neural network (ANN)-based models for joint prediction of the Newtonian and nonNewtonian viscosities of black liquor over wide ranges of solids concentration, temperature and shear rate. Performance evaluation and consideration for network's complexity show that a simple multi-layer feed-forward [7, 1] network with strong generalization capability and having $R^{2}=1.0$ and maximum absolute relative error (MARE $=8 \%$ ) between actual and predicted data, can successfully be used in place of multiple functional models for the analysis of black liquor viscosity behaviour over a wide range of conditions. During training, the network experienced learning difficulties; this was possibly due to redundancy of shear rate factor when the liquor exhibits pure Newtonian behaviour. A network with internal classification may be able to eliminate this difficulty.

\section{REFERENCES}

[1] A. Moosavifar, M. Sedin, and H. Theliander, "Correlations for the viscosity of lignin lean black liquor," Nordic Pulp \& Paper Research Journal, vol. 25, no. 1, pp. 15-20, 2010.

[2] A. Moosavifar, P. Sedin, and H. Theliander, "Viscosity and boiling point elevation of black liquor: Consequences when lignin is extracted from the black liquor," Nordic Pulp and Paper Research Journal, vol. 21, no. 2, pp. 180-187, 2006.

[3] V. Venkatesh and X. N. Nguyen, "Evaporation and concentration of black liquor," in Chemical recovery in the alkaline pulping processes, R. P. Green and G. Hough, Eds. pp. 5-33, Atlanta, GA: TAPPI Press, 1992.

[4] S. B. Alabi, C. J. Williamson, and J. Lee, "Non-Newtonian behaviour of black liquors: A case study of the Carter Holt Harvey Kinleith mill liquor," Appita Journal, vol. 65, no. 1, pp. 63-70, 2012.

[5] T. N. Adams and J. W. Frederick, Kraft Recovery Boiler Physical and Chemical Processes, NY: The American Paper Institute, Inc, 1988.

[6] J. W. Frederick, "Black liquor properties," in Kraft Recovery Boilers, T. N. Adams, Ed. pp. 59-100, Atlanta, GA: TAPPI Press, 1997.

[7] A. K. Ghosh, "Generalised correlation of viscosity of concentrated black liquor and its application to droplet size distribution," in Proc. APCChE and CHEMECA '93, Melbourne, Australia, pp. 233-238, 1993.

[8] E. Vakkilainen, "Chemical recovery, Chemical pulping," Papermaking Science and Technology, J. Gullichsen and C.-J. Fogelholm, Eds. pp. B6-B34, Helsinki, Finland: Fapet Oy, 1999.

[9] S. B. Alabi, C. J. Williamson, and J. Lee, "Viscosity models for New Zealand black liquor at low solids concentrations," Asia-Pacific Journal of Chemical Engineering, vol. 5, no. 4, pp. 619-625, 2010.

[10] S. B. Alabi and C. J. Williamson, "Artificial neural network-based prediction of the newtonian viscosity of black liquor over a wide range of process conditions," in Proc. CHEMECA 2013, Brisbane, Australia, 2013.

[11] A. A. Zaman and A. L. Fricke, "Steady shear flow properties of high solids softwood kraft black liquors: effects of temperature, solids concentrations, lignin molecular weight and shear rate," Chem. Eng. Comm, vol. 139, pp. 201-221, 1995.

[12] A. A. Zaman and A. L. Fricke, "Kraft black liquor rhelogical behaviour with respect to solids concentration, temperature, and shear rate," AIChE Symposium Series, 1995, pp. 162-171.

[13] European Commission, Integrated Pollution Prevention and Control (IPPC).Reference Document on Best Available Techniques in the Pulp and Paper Industry, 2001.

[14] ThermExcel. (2013). Properties of fluids-physical characteristics of water. [Online]. http://www.thermexcel.com/english/tables/eau_atm.htm

[15] S. N. Bhattacharya, Rheology Fundamentals and Measurements, Melbourne, Australia: Royal Melbourne Institute of Technology, 2002.

[16] M. M. Cross, "Rheology of non-Newtonian fluids: A new flow equation for pseudoplastic systems," Journal of Colloid Science, vol. 20, no. 5, pp. 417-437, 1965 
[17] A. A. Zaman and A. L. Fricke, "Newtonian viscosity of high solids kraft black liquors: effects of temperature and solids concentrations," Industrial and Engineering Chemistry Research, vol. 33, no. 2, pp. 428-435, 1994.

[18] N. Cunningham. (2010). Making use of models: the Cross model. [Online]. Available: http://www.rheologyschool.com/id46.html

[19] H. Demuth, M. Beale, and Hagan, M., Neural network toolbox ${ }^{T M} 6$ user's guide, The MathWorks Inc, Nattick,MA, 2008.

[20] Statsoft Inc. (2010). Electronic statistics textbook. [Online]. Available: http://www.statsoft.com/textbook/

[21] R. P. Chhabra and J. F. Richardson., Non-Newetonian flow and applied rheology (Engineering applications), 2nd ed., UK: Elsevier Ltd, 2008.

[22] A. Abraham, "Artificial neural networks," in Handbook for Measurement Systems Design, P. Sydenham and R. Thorn Eds., pp. 901-908, London John Wiley and Sons Ltd., 2005.

[23] S. A. Rounds, " Development of a neural network model for dissolved oxygen in the Tualatin river, Oregon," in Proc. Second Federal Interagency Hydrological Modeling Conference, Las Vegas, Nevada, 2002.

[24] A. B. Bulsari, Neural Networks for Chemical Engineers, Amsterdam: Elsevier, 1995,

[25] M. Smith, Neural Networks for Statistical Modeling, Boston, MA: International Thompson Computer Press, 1996, p. 251.

[26] S. B. Alabi and C. J. Williamson, "Centrifugal pump-based predictive models for Kraft Black Liquor Viscosity: An artificial neural network approach," Industrial \& Engineering Chemistry Research, vol. 50, no. 17, pp. 10320-10328, 2011
[27] (2010). NIST/SEMATECH e-Handbook of Statistical Methods [Online]. Available: http://www.itl.nist.gov/div898/handbook/, NIST/SEMATECH

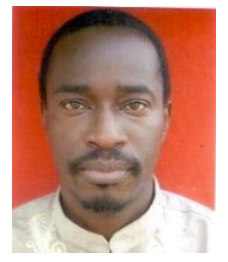

Sunday Boladale Alabi holds B.Sc. and M.Sc degree in chemical engineering from the Obafemi Awolowo University, Ile-Ife, Nigeria. He holds a $\mathrm{Ph} . \mathrm{D}$. degree in chemical and process engineering from the University of Canterbury, Christchurch, New Zealand. $\mathrm{He}$ is a lecturer in the Department of Chemical and Petroleum Engineering, University of Uyo, Uyo, Akwa Ibom, Nigeria. His research interests include process modelling, control and optimization, soft sensing and rheology of complex fluids. He is a member of the Nigerian Society of Chemical Engineers (MNSChE).

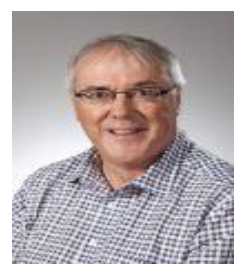

Chris Williamson holds B.Eng. and Ph.D. degree in chemical and process engineering from the University of Canterbury, New Zealand. He is a senior lecturer in chemical and process engineering at the University of Canterbury, Christchurch, New Zealand. He has lectured on thermodynamics, process control and process engineering design. His recent research interests include process control and measurement, including applications in the pulp and paper industry, and renewable fuels production from biomass via the Fischer-Tropsch process. 
